\title{
Primary breast angiosarcoma with disseminated intravascular coagulation is successfully treated with self-subcutaneous unfractionated heparin calcium injection: A case report
}

\author{
TOSHINARI YAGI ${ }^{1}$, HARUMI NAKAMURA ${ }^{2}$, TORU WAKAMATSU ${ }^{3}$, YOSHINORI IMURA $^{3}$, \\ HIRONARI TAMIYA ${ }^{3}$, HIDEAKI SABE ${ }^{3}$, KATSUNARI YAMASHITA ${ }^{3}$, \\ MAKIYO WATANABE ${ }^{3}$ and SATOSHI TAKENAKA ${ }^{3}$ \\ Departments of ${ }^{1}$ Outpatient Chemotherapy, ${ }^{2}$ Diagnostic Pathology and Cytology, and \\ ${ }^{3}$ Orthopedic Surgery, Osaka International Cancer Institute, Osaka 541-8567, Japan
}

Received November 24, 2020; Accepted February 26, 2021

DOI: $10.3892 / \mathrm{mco} .2021 .2266$

\begin{abstract}
Angiosarcoma is a rare sarcoma with a poor prognosis and is prone to disseminated intravascular coagulation (DIC), where DIC often interferes with chemotherapy. Primary angiosarcoma of the breast (PASB) is a type of angiosarcoma that is located in mammary parenchyma and is not associated with radiation exposure. The current study reported a 47-year-old female with DIC associated with PASB. The DIC of the patient relapsed during mono-chemotherapy with paclitaxel (PTX) after first-line anticoagulant therapy using thrombomodulin- $\alpha$. The second-line danaparoid sodium therapy, followed by self-subcutaneous injection of unfractionated heparin calcium (UFH), resulted in long-term stabilization of DIC. Under this second-line anticoagulant therapy, the patient continued chemotherapy and chemoradiotherapy for $>13$ months in the outpatient setting without impairment of quality of life. The present case suggested that self-subcutaneous injections of UFH may be a useful therapeutic option for long-term control of DIC associated with PASB. However, further prospective clinical trails are needed to verify the efficacy of self-subcutaneous injection of UFH in similar settings.
\end{abstract}

Correspondence to: Dr Toshinari Yagi, Department of Outpatient Chemotherapy, Osaka International Cancer Institute, 3-1-69 Otemae, Chuo-ku, Osaka 541-8567, Japan

E-mail: yagi-to@mc.pref.osaka.jp

Abbreviations: DIC, disseminated intravascular coagulation; PASB, primary angiosarcoma of the breast; UFH, unfractionated heparin calcium; CT, computed tomography; NR, normal range; PT-INR, prothrombin time-international normalized ratio; APTT, activated partial thromboplastin time; $\mathrm{CD}$, cluster of differentiation; ERG, erythroblast transformation-specific (ETS)-related gene; PTX, paclitaxel

Key words: angiosarcoma, disseminated intravascular coagulation, heparin

\section{Introduction}

Disseminated intravascular coagulation (DIC) is a well-known hemostatic complication of solid tumors. It is usually identified at the time of presentation and can sometimes cause excessive bleeding or thromboembolic complication. In comparison, primary angiosarcoma of the breast (PASB) is a malignant primary endothelial neoplasm of mammary parenchyma that is not associated with radiation exposure. The median age of patients at diagnosis is 40 years, compared with a median age of 70 years for the secondary angiosarcoma (1). Adem et al (2) reported that angiosarcoma accounts for one-fourth of all breast sarcomas, excluding metaplastic carcinomas and phyllodes tumors. D'Angelo et al (3) performed a retrospective analysis of 119 patients with metastatic angiosarcoma, $24 \%$ of which had radiation-associated angiosarcoma. The most frequent primary sites were the chest wall/breast $(37.31 \%)$, viscera (22\%), and head/neck (20\%). The median overall survival of patients with metastatic angiosarcoma with origin at all sites was 12.1 months. Even if DIC is a common complication associated with angiosarcomas and often interferes with patients' chemotherapy (4), there have been few case reports on anticoagulant therapies for DIC associated with angiosarcoma because angiosarcoma is rare. In the current study, we present a rare case of a PASB with DIC that was controllable by a self-subcutaneous injection of unfractionated heparin calcium (UFH).

\section{Case report}

In November 2019, a 47-year-old woman with a breast tumor and multiple lung and bone metastases was referred to our hospital for further diagnosis and treatment. She had consulted a nearby hospital complaining of swelling of the left breast in July 2018. Imaging examinations revealed a giant mass in her breast. Core needle biopsy was performed, resulting in the histological diagnosis of hemangioma. Lumpectomy was performed in September 2018. In August 2019, her left nipple became swollen, and computed tomography (CT) revealed bilateral multiple lung and bone metastases (Fig. 1). 
Table I. Summary of laboratory data.

\begin{tabular}{|c|c|c|c|}
\hline Parameter & Normal range & 1st Admission & 2nd Admission \\
\hline \multicolumn{4}{|l|}{ Complete blood count } \\
\hline White blood cells (x109/1) & $3.30-8.60$ & 4.67 & 3.35 \\
\hline Hemoglobin (g/dl) & $11.6-14.8$ & 11.3 & 9.3 \\
\hline Platelets (x109/1) & $158-348$ & 79 & 52 \\
\hline \multicolumn{4}{|l|}{ Biochemistry } \\
\hline Aspartate aminotransferase (U/l) & $13-30$ & 17 & 23 \\
\hline Alanine aminotransferase (U/l) & $7-23$ & 9 & 22 \\
\hline Lactate dehydrogenase (U/l) & $124-222$ & 323 & 433 \\
\hline Total bilirubin (mg/dl) & $0.4-1.5$ & 0.6 & 0.4 \\
\hline Blood urea nitrogen (mg/dl) & $8.0-20.0$ & 15.0 & 12.0 \\
\hline Creatinine (mg/dl) & $0.47-0.79$ & 0.64 & 0.51 \\
\hline Total protein $(\mathrm{g} / \mathrm{dl})$ & $6.6-8.1$ & 7.9 & 6.7 \\
\hline C-reactive protein (mg/dl) & $<0.14$ & 0.14 & 0.09 \\
\hline \multicolumn{4}{|l|}{ Coagulation } \\
\hline PT-INR & $0.85-1.20$ & 1.12 & 1.22 \\
\hline APTT (sec) & $24.0-39.0$ & 31.8 & 30.9 \\
\hline AT-3 (\%) & $75-130$ & 100 & 100 \\
\hline Fibrinogen (mg/dl) & $200-400$ & 127 & 84 \\
\hline $\mathrm{FDP}(\mu \mathrm{g} / \mathrm{ml})$ & $<5.0$ & 297.9 & No data \\
\hline D-dimer $(\mu \mathrm{g} / \mathrm{ml})$ & $<1.0$ & 112.9 & 215.8 \\
\hline
\end{tabular}

PT-INR, prothrombin time-international normalized ratio; APTT, activated partial thromboplastin time; AT-3, antithrombin-3; FDP, fibrinogen/fibrin degradation product.

At this point, a malignant tumor was suspected and she was transferred to our hospital.

She was admitted to our hospital in November 2019. Upon admission, a red mass that was $3 \mathrm{~cm}$ in diameter was observed on the left nipple during physical examination. Whole body magnetic resonance imaging revealed multiple bone metastases in the vertebra, sternum, ilium, bilateral humeri, and bilateral femurs. Laboratory tests yielded the following results (Table I): White blood cells $=4.67 \times 10^{9} / 1$, hemoglobin $=11.3 \mathrm{~g} / \mathrm{dl}$, platelet count $=79 \times 10^{9} / 1$ [normal range (NR): $158-348 \times 10^{9} / 1$ ], total bilirubin=0.6 $\mathrm{mg} / \mathrm{dl}$, aspartate aminotransferase $=17 \mathrm{U} / 1$, alanine aminotransferase $=9 \mathrm{U} / 1$, lactate dehydrogenase=323 U/1 (NR: 124-222 U/1), blood urea nitrogen $=15 \mathrm{mg} / \mathrm{dl}$, creatinine $=0.64 \mathrm{mg} / \mathrm{dl}$, prothrombin time-international normalized ratio $(\mathrm{PT}-\mathrm{INR})=1.12$, activated partial thromboplastin time $($ APTT $)=31.8 \mathrm{sec}$, fibrinogen=127 mg/dl (NR: 200-400 mg/dl), fibrinogen/fibrin degradation product $=297.9 \mu \mathrm{g} / \mathrm{ml}(\mathrm{NR}<5.0 \mu \mathrm{g} / \mathrm{ml})$, and D-dimer $=112.9 \mu \mathrm{g} / \mathrm{ml}(\mathrm{NR}<1.0 \mu \mathrm{g} / \mathrm{ml})$. Her laboratory data met the diagnostic criteria (6 points) for DIC from the Japanese Society on Thrombosis and Hemostasis (5). However, there were no signs of deep vein thrombosis or pulmonary embolism, or signs of bleeding tendency such as subcutaneous bleeding or mucosal bleeding.

Re-examination of the resected left breast tumor by our hospital pathologists resulted in the diagnosis of high-grade angiosarcoma, as the mass was mainly composed of atypically anastomosing vessels that spread invasively, and dense solid foci containing oval or spindle cells with nuclear enlargement and hyperchromasia (Fig. 2). Tumor cells were immunohistochemically positive for cluster of differentiation (CD)34, CD31, and erythroblast transformation-specific [ETS]-related gene (ERG). The immunohistochemistry was performed using monoclonal antibodies for CD34 (clone QBEnd/10, cat. no. 518-102418, Roche), CD31 (clone JC70, cat. no. 518-103231, Roche), and ERG (clone EPR3864, cat. no. 518-110819, Roche). The immunostaining was performed using a Ventana BenchMark ULTRA IHC/ISH Staining Module ${ }^{\circledR}$. Antigen retrieval was performed by placing tissue sections in ULTRA CC1 buffer ( $\mathrm{pH} 9.0)$ for the optimum amount of time (32-64 min). Subsequently, slides were incubated at $37^{\circ} \mathrm{C}$ for varying amounts of time: $8 \mathrm{~min}$ for CD34, $32 \mathrm{~min}$ for CD31, and $24 \mathrm{~min}$ for ERG. The OptiVIEW DAB Detection Kit (Ventana Medical Systems, Inc.) was used to detect the mouse $\operatorname{IgG}$, mouse IgM, and rabbit primary antibodies. Slides were then counterstained with hematoxylin.

Thrombomodulin- $\alpha(380 \mathrm{U} / \mathrm{kg})$ was started on the day of admission and lasted for 7 days. Weekly PTX infusion $\left(100 \mathrm{mg} / \mathrm{m}^{2}\right)$ was started on the second day. Her hemostasis test improved and she was discharged 13 days after admission. However, she was hospitalized a second time 3 days after discharge because her DIC relapsed.

On her second admission, hemostasis tests yielded the following results: Platelet count $=52 \times 10^{9} / 1$, PT-INR $=1.22$, APTT $=30.9 \mathrm{sec}$, fibrinogen $=84 \mathrm{mg} / \mathrm{dl}$, and D-dimer $=$ $215.8 \mu \mathrm{g} / \mathrm{ml}$. At this point, we decided that angiosarcoma activity and DIC were uncontrollable by mono-chemotherapy 

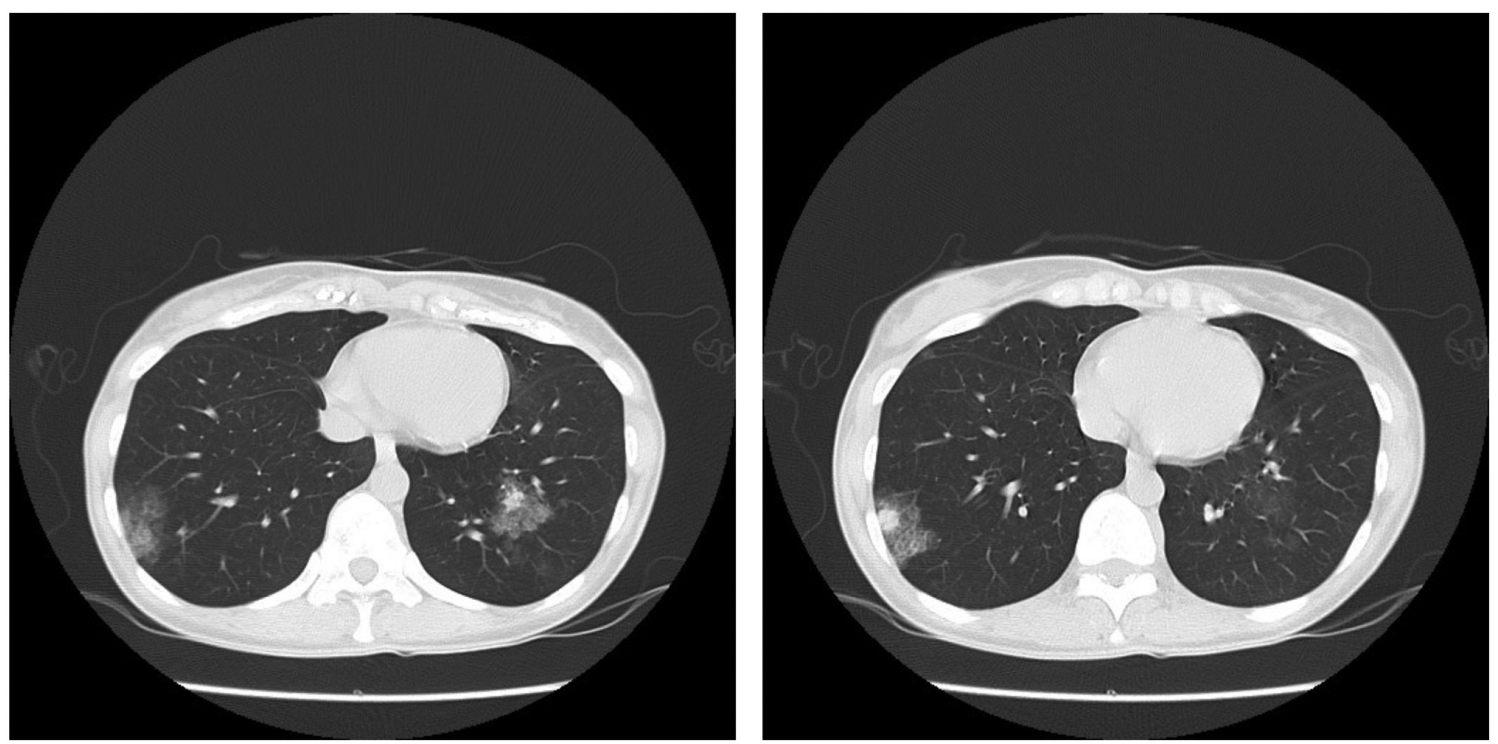

Figure 1. Computed tomography scan performed at the previous hospital (Osaka Breast Clinic) showing multiple core nodules with a ground glass appearance, alongside suspected metastatic lesions and reactive changes or pulmonary hemorrhage.

of PTX. Therefore, to control DIC, danaparoid sodium $(1,250$ units, every $12 \mathrm{~h})$ was infused for 13 days, and chemotherapy was switched from PTX to eribulin mesylate $\left(1.4 \mathrm{mg} / \mathrm{m}^{2}\right.$; infusions performed on days 1 and 8 , of every 21-day cycle). After treatment with danaparoid sodium was started, her DIC had improved. For outpatient treatment, infusion of danaparoid sodium was switched to self-subcutaneous injection of UFH (5,000 units, once every $12 \mathrm{~h})$. After this anticoagulant change, her DIC remained controllable, and eribulin mesylate was continued for 2 months (Fig. 3).

CT in February 2020 revealed an increase in the left breast mass, whereas lung metastatic lesions became reduced. Eribulin mesylate was stopped and chemoradiotherapy was performed, which consisted of intensity-modulated radiotherapy (total $60 \mathrm{~Gy} / 30$ fraction) and concurrent weekly PTX infusions $\left(63 \mathrm{mg} / \mathrm{m}^{2}\right)$, as well as self-subcutaneous injections of UFH. PTX was re-started because a previous report suggested that chemoradiotherapy with taxane and maintenance chemotherapy in cutaneous angiosarcoma were efficacious (6), but there have been no reports of eribulin mesylate with radiotherapy.

After the radiotherapy,PTX $\left(63 \mathrm{mg} / \mathrm{m}^{2}\right.$; infusions performed on days 1,8 , and 15 of every 28 -day cycle) was continued as a maintenance therapy for 6 months. In November 2020, multiple liver metastases were diagnosed by follow-up CT, but her DIC remained stable, and PTX was replaced by eribulin mesylate $\left(1.1 \mathrm{mg} / \mathrm{m}^{2}\right.$; infusions performed on days 1 , day 8 , of every 21-day cycle) again. At the time of reporting, the patient has been continuing the self-subcutaneous injections of UFH as well as eribulin mesylate infusions. No adverse events associated with UFH use have been observed. Her PASB remained in stable disease, whereas DIC became controllable.

\section{Discussion}

DIC is a common complication associated with angiosarcoma and often interferes with a patient's chemotherapy.
Farid et al (4) retrospectively reviewed case records of 42 patients diagnosed with angiosarcoma at Mount Sinai Hospital, wherein 7 patients (17\%) met clinical criteria for DIC. In their report, all patients who received systemic antineoplastic therapy with resultant disease response or stability had their DIC resolved in tandem with clinical improvement. DIC also recurred at the time of disease progression in all cases. Only a few case reports mention anticoagulant therapies for DIC associated with angiosarcoma. Honda et al (7) reported the first case of a patient having primary cardiac angiosarcoma with coexisting DIC who was successfully treated with nab-PTX. This patient's DIC, treated with transfusion of fresh-frozen plasma and recombinant thrombomodulin- $\alpha$ (used for 6 days), improved shortly after the initiation of chemotherapy. Rosen et al (8) reported a case of hepatic angiosarcoma with a primary focus on the management of the patient's DIC. In their report, the patient's DIC became controlled after initial treatment with unfractionated heparin and subsequent enoxaparin treatment.

In our study, the patient's DIC improved through chemotherapy with PTX and recombinant thrombomodulin- $\alpha$. However, it re-exacerbated soon after recombinant thrombomodulin- $\alpha$ was completed. As a second treatment for DIC, danaparoid sodium was used and her DIC improved again. To switch from inpatient treatment to outpatient treatment, infusion of danaparoid sodium was switched to self-subcutaneous injections of UFH. This UFH resulted in long-term stabilization of DIC ( $>13$ months) and made the continuity of chemotherapy and chemoradiotherapy possible as an outpatient treatment.

Based on the degree of fibrinolytic activation, there are diverse subtypes in DIC, such as suppressed-fibrinolytic-type, balanced-fibrinolytic-type, and enhanced-fibrinolytic-type (9). Our patient's laboratory findings, that was measured under the treatment of UFT, showed a marked elevation in both thrombin-antithrombin complex (more than $120.0 \mathrm{ng} / \mathrm{ml}: \mathrm{NR}<4 \mathrm{ng} / \mathrm{ml}$ ) and plasmin- $\alpha 2$ plasmin complex 
A

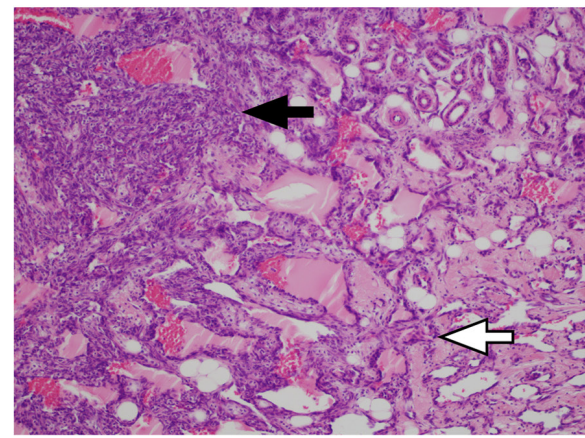

C

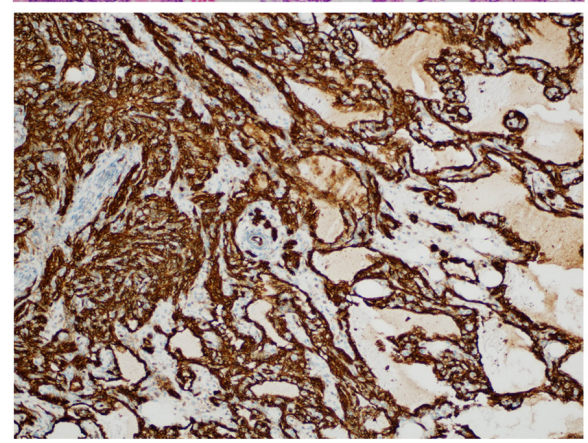

B

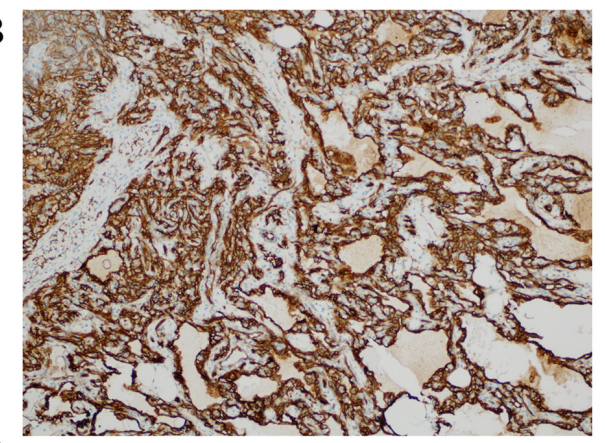

D

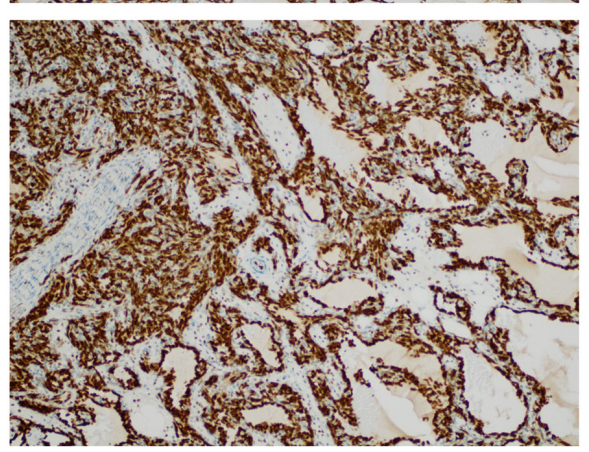

Figure 2. Microscopic features of the angiosarcoma of the left breast resected at the previous hospital (Osaka Breast Clinic). (A) The mass was mainly composed of atypically anastomosing vessels that spread invasively (white arrow; hematoxylin and eosin staining; magnification, x100) and dense solid foci containing oval or spindle cells with nuclear enlargement and hyperchromasia (black arrow). Tumor cells were immunohistochemically positive for (B) CD34 (magnification, x100), (C) CD31 (magnification, x100) and (D) ERG (magnification, x100). ERG, erythroblast transformation-specific (ETS)-related gene.

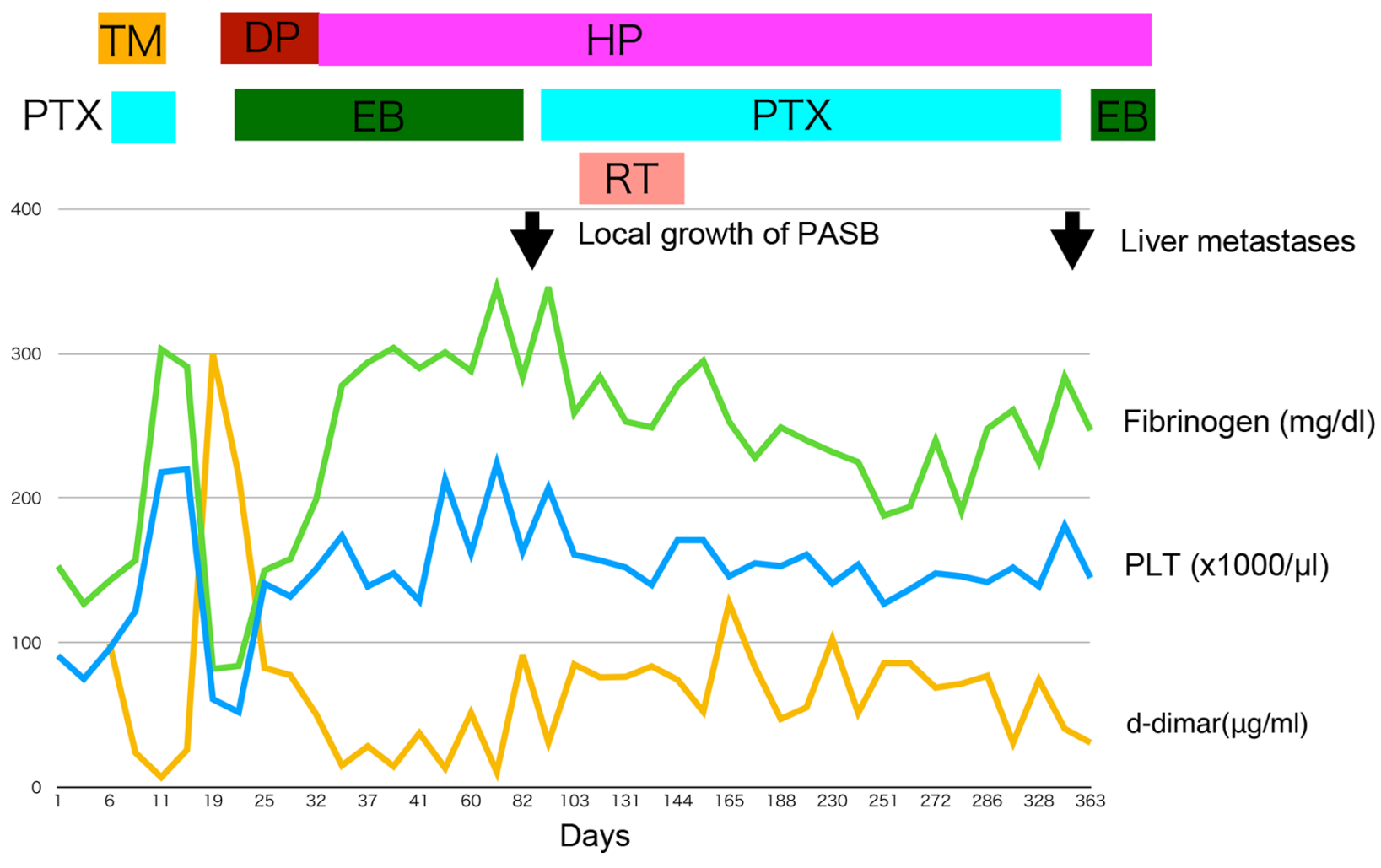

Figure 3. Clinical course of the patient. TM, thrombomodulin- $\alpha$; DP, danaparoid sodium; HP, unfractionated heparin sodium; PTX, paclitaxel; EB, eribulin mesylate; RT, radiotherapy; PASB, primary angiosarcoma of the breast; PLT, platelet count.

$(8.5 \mu \mathrm{g} / \mathrm{ml}$ : NR 0-0.8 $\mu \mathrm{g} / \mathrm{ml})$. We speculated that our patient had enhanced-fibrinolytic-type DIC. For this type of DIC, combination therapy with heparin and tranexamic acid has been reported to be very effective (9). In our case, addition of tranexamic acid was not considered because there was no bleeding symptom, and her DIC was in a stable condition at 
the time of UFH start. The mean survival time in metastatic disease was 6.4 months (range: 1-11 months) in 7 cases of angiosarcoma with DIC reported by Farid et al (4). Therefore, we speculate that the use of UFH helped prolong our patient's life. Mono-chemotherapy with PTX after first-line anticoagulant therapy with recombinant thrombomodulin- $\alpha$ could not stabilize DIC, but combination therapy of PTX and UFT after chemoradiotherapy could. So, we considered that UFH, rather than PTX, contributed to the stabilization of her DIC.

Self-subcutaneous injection of heparin is effective for chronic DIC associated with aortic aneurysm and vascular malformations, and is sometimes indicated for these patients $(10,11)$. However, there have been few case reports where it was used to treat DIC with malignancy. Some studies on cancers other than angiosarcoma reported results similar to ours. Makari et al (12) reported a case of gastric cancer with Trousseau's syndrome and pulmonary embolism treated with S-1 while receiving subcutaneous injections of heparin. Odani et al (13) reported a case of Trousseau's syndrome caused by clear cell adenocarcinoma of the ovary, wherein the patient received four courses of PTX and carboplatin chemotherapy while receiving subcutaneous injections of unfractionated heparin.

In Japan, many oncologists tend to try first-line chemotherapy with anticoagulant therapy for solid tumors with DIC. However, once the patient's DIC relapses, if there is no second-line chemotherapy with a high response rate, they often decide that the tumors, as well as DIC, are beyond control and hence give up on aggressive treatments. Our case suggests that if self-subcutaneous injections of UFH are used, some patients with PASB complicated by DIC can continue to receive chemotherapies or chemoradiotherapies in the outpatient setting without impairing quality of life. Nevertheless, we would like to acknowledge a limitation in this case report. Including our case, there have been few case reports on DIC associated with malignancies treated by self-subcutaneous injection of UFH. DIC associated with more aggressive PASB may not be controllable by self-subcutaneous injections of UFH. This study is based on only one case report; therefore, further prospective investigations are needed to elucidate the clinical efficacy of self-subcutaneous injections of UFH for the treatment of PASB with DIC.

In summary, we reported the case of a woman with PASB complicated by DIC. The DIC relapsed after the first line anticoagulant therapy of thrombomodulin- $\alpha$, but the second line danaparoid sodium followed by self-subcutaneous injection of UFH resulted in long-term stabilization of the DIC. Our case suggests that if self-subcutaneous injections of UFH are used, some patients diagnosed with PASB with DIC can continue to receive chemotherapies or chemoradiotherapies in the outpatient setting without having their quality of life impaired. Nevertheless, further prospective clinical trials are needed to verify the efficacy of self-subcutaneous injection of UFH in similar settings.

\section{Acknowledgements}

The authors would like to thank Mr. Kikuichi Nakagawa for his technical assistance.

\section{Funding}

No funding was received.

\section{Availability of data and materials}

The datasets used and/or analyzed during the present study are available from the corresponding author on reasonable request.

\section{Authors' contributions}

TY conceived the present study. HN pathologically diagnosed the patient and revised the manuscript. TW, YI, HT, HS, KY, MW and ST collected clinical data. All authors read and approved the final manuscript prior to submission. TY and ST confirm the authenticity of all the raw data.

\section{Ethics approval and consent to participate}

The soft tissue mass of the patients was collected after written informed consent was obtained from the patient according to the protocol approved by the Osaka International Cancer Institute (Osaka, Japan).

\section{Patient consent for publication}

Written informed consent was obtained from the patient for the publication of patient data and associated images.

\section{Competing interests}

The authors declare that they have no competing interests.

\section{References}

1. Antonescu CR, Hunt KK, Rowe JJ and Thway K: Primary angiosarcoma of the breast. In: Breast Tumours. 5th edition. WHO Classification of Tumours Editorial Board (ed.) International Agency for Research on Cancer, Lyon, pp200-201, 2019.

2. Adem C, Reynolds C, Ingle JN and Nascimento AG: Primary breast sarcoma: Clinicopathologic series from the Mayo clinic and review of the literature. Br J Cancer 91: 237-241, 2004.

3. D'Angelo SP, Munhoz RR, Kuk D, Landa J, Hartley E, Bonafede M, Dickson MA, Gounder M, Keohan ML, Crago AM, et al: Outcomes of systemic therapy for patients with metastatic angiosarcoma. Oncology 89: 205-214, 2015.

4. Farid M, Ahn L, Brohl A, Cioffi A and Maki RG: Consumptive coagulopathy in angiosarcoma: A recurrent phenomenon? Sarcoma 2014: 617102, 2014.

5. Asakura H, Takahashi H, Uchiyama T, Eguchi Y, Okamoto K, Kawasugi K, Kobayashi T, Taki M, Tujinaka T, et al: Diagnostic criteria for DIC from the Japanese Society on Thrombosis and Hemostasis-2017 edition. Jpn J Thromb Hemost 28: 369-391, 2017 (In Japanese).

6. Fujisawa Y, Yoshino K, Kadono T, Miyagawa T, Nakamura Y and Fujimoto M: Chemoradiotherapy with taxane is superior to conventional surgery and radiotherapy in the management of cutaneous angiosarcoma: A multicentre, retrospective study. Br J Dermatol 171: 1493-1500, 2014.

7. Honda K, Ando M, Sugiyama K, Mitani S, Masuishi T, Narita Y, Taniguchi H, Kadowaki S, Ura T and Muro K: Successful treatment of cardiac angiosarcoma associated with disseminated intravascular coagulation with nab-paclitaxel: A case report and review of the literature. Case Rep Oncol 10: 863-870, 2017.

8. Rosen EA, Vallurupalli M, Choy E, Lennerz JK and Kuter DJ: Management of disseminated intravascular coagulation in a patient with hepatic angiosarcoma: A case report. Medicine (Baltimore) 97: e13321, 2018. 
9. Asakura $\mathrm{H}$ : Classifying types of disseminated intravascular coagulation: Clinical and animal models. J Intensive Care 2: 20, 2014.

10. Miyahara S, Yasu T, Yamada Y, Kobayashi N, Saito M and Momomura S: Subcutaneous injection of heparin calcium controls chronic disseminated intravascular coagulation associated with inoperable dissecting aortic aneurysm in an outpatient clinic. Intern Med 46: 727-732, 2007.

11. Yamada S and Asakura H: Management of disseminated intravascular coagulation associated with aortic aneurysm and vascular malformations. Int J Hematol 113: 15-23, 2021.

12. Makari Y, Nagase H, Iijima S, Miyo M, Sakamoto T, Doi T, Hoshi M, Miyake Y, Oshima S, Ikeda K, et al: A case of advanced gastric cancer with Trousseau syndrome and pulmonary embolism. Gan To Kagaku Ryoho 39: 2363-2365, 2012 (In Japanese).
13. Odani N, Ono T, Kusumoto A, Sanada Y, Oda H, Tanabe R, Nishimura M, Takikawa K, Matsumoto K, et al: A case of Trousseau's syndrome: Multiple cerebral infarction caused by clear cell adenocarcinoma of the ovary. Tokyo Sanka Fujinka Gakkai Zasshi 64: 509-514, 2015 (In Japanese).

(i)(2) This work is licensed under a Creative Commons c) AY NO Attribution-NonCommercial-NoDerivatives 4.0 International (CC BY-NC-ND 4.0) License. 